\section{COMPUTATIONAL ECOLOGY}

\section{The eyes have it}

Proc. Natl Acad. Sci. USA http://doi.org/b4jh (2017)

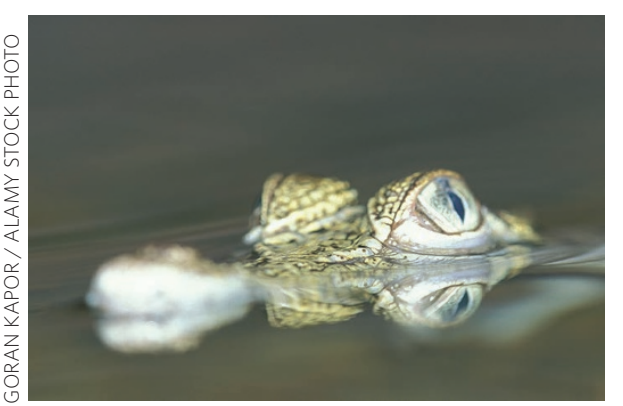

Try to picture our ancestors' emergence from the ocean's depths and you're guaranteed to imagine fins morphing into limbs. But research from Malcolm MacIver and colleagues indicates that a different change may have preceded our legged conversion. MacIver et al. examined the eye-socket sizes of animals spanning the transition from water to land, occurring some 385 million years ago. They found that a three-fold increase in socket size - which is highly correlated with eye and pupil size predated the completion of the shift to limbs.

The authors computed four measures of visual function for the mean pupil size of finned and digited groups, estimated from their socket size. They calculated the visual ranges in aquatic and above-water environments using a simple model, and found that the tripling of eye size translated to a negligible performance increase for animals remaining underwater. But for animals capable of looking out to the horizon, the increase was found to be more than a million times larger. The information increase afforded by long-range vision may have prompted our evolution to terrestriality.

\section{QUANTUM PHYSICS}

\section{Heroes of zeroes}

Phys. Rev. Lett. (in the press); preprint at http://arxiv.org/abs/1608.03679

More than one hundred years ago, German mathematician Bernhard Riemann proposed a conjecture about the nontrivial zeroes of the Riemann zeta function: they all lie on a line in the complex plane that has a real part of $1 / 2$. The Riemann hypothesis, as it's known, features on the list of 23 unsolved problems that David Hilbert published in 1900. Proving it would turn hundreds of mathematical statements into bona fide theorems - but a rigorous proof is still missing.

Carl Bender and colleagues have paved the way to a possible solution by exploiting a connection with physics. The Hilbert-Pólya conjecture supposes the existence of a Hamiltonian whose eigenvalues correspond to the imaginary parts of the nontrivial zeroes of the zeta function. Bender et al. constructed an operator that plays the role of this Hamiltonian. And with further analysis of the properties of this Hamiltonian, physics may yet shed light on the Riemann hypothesis, bringing new insights into this century-old mathematical problem.

\section{ACCRETION}

\section{Gone with the wind}

Nat. Astron. 1, 0062 (2017)

Much like other cosmic beasts, black holes grow by accreting mass from a surrounding disk. And they are messy eaters. To

conserve the disk's angular momentum and prolong the feast, they eject ionized gas in $A K$ a wide fan outside the accretion disk plane.
ACTIVE SOFT MATTER

\section{Toroidal swimmers}

The chemical interactions between suspended colloidal particles and a solute can induce the particles to self-propel, a phenomenon known as autophoresis. Studies of autophoretic motion have so far been restricted to spheroidal colloids. Now, Lasse Schmieding and colleagues have performed a theoretical analysis of the autophoresis of toroidal colloidal particles - a torus being the archetype of a shape that is not simply connected.

The authors first considered the axisymmetric case, and adopted a continuum approach for modelling the flow field of the torus-solute system. Chemical interactions were accounted for by two quantities: surface chemistry and surface mobility. When these were assumed to be constant, the torus did not self-propel. However, concentration gradients from the torus's hole generated a pumping flow.

Proper autophoresis was obtained for a Janus torus, for which the two 'sides' have different surface chemistries and mobilities. Schmieding etal. found that the Janus torus claiming the highest swimming speed was characterized by a relatively small central hole.
These plasma outflows, or black hole winds, leave a mark in the absorption lines of the $\mathrm{X}$-ray spectra, but the exact mechanism responsible for launching the winds is not clear. Using magnetohydrodynamics simulations, Keigo Fukumura and colleagues have now found compelling reasons to point the finger at a likely culprit: the magnetic field.

Black hole winds of magnetic origin are expected to be very fast (up to $0.2 c$ ). Fukumura and co-workers previously used magnetohydrodynamic wind models to explain the properties of such ultrafast outflows in the X-ray spectra of supermassive black holes, giving weight to the magnetic origin scenario. Now Fukumura et al. have extended their approach and found that it works equally well for a stellar-mass black hole. This suggests that the magnetic origin of black hole winds is a universal feature across the mass spectrum.

\section{GENERAL RELATIVITY}

\section{Going underground}

Rev. Sci. Instrum. 88, 034502 (2017)

In 1919 a total solar eclipse allowed Arthur Eddington to put Einstein's general theory of relativity to the test to see if the Sun's mass was really warping spacetime, deflecting the light from faraway stars. A team of scientists is now looking for general relativitistic phenomena using not the Sun, but the Earth - and adding rotational effects into the mix.

Using ring laser gyroscopes essentially optical interferometers placed in a ring configuration - Jacopo Belfi and colleagues are hoping to detect a gravitomagnetic effect known as the Lense-Thirring effect. Arising from the Earth's mass and angular momentum, this general relativitistic phenomenon should cause a difference between the rotation rate of the Earth measured by a ground-based observatory and the value measured in an inertial reference frame.

In order to achieve the resolution required to see such effects, the team have gone deep underground in the international laboratory of Gran Sasso in central Italy, far away from external disturbances. And they have now made an important step towards their goal by demonstrating a pilot prototype, known as GINGERino, which can make Earth rotation measurements, as well as act as a seismic observatory.

Written by Luke Fleet, lulia Georgescu, Abigail Klopper, Yun Li and Bart Verberck. 